\title{
Atacama Large Millimeter/Submillimeter Array (ALMA)
}

\author{
Jean L. Turner ${ }^{1}$ and H. Alwyn Wootten ${ }^{2} \dagger$ \\ ${ }^{1}$ Department of Physics and Astronomy, UCLA, Los Angeles, CA 90095-1547, USA \\ email: turner@astro.ucla.edu \\ ${ }^{2}$ NRAO, 520 Edgemont Road, Charlottesville, VA 22903-2475, USA \\ email: awootten@nrao.edu
}

\begin{abstract}
The Atacama Large Millimeter/Submillimeter Array (ALMA) is an international effort to construct an instrument capable of matching the exquisite imaging properties of optical space telescopes at millimeter and submillimeter wavelengths. ALMA science will transform our vision of the cold, dusty, and gaseous universe, from extrasolar planets to the youngest galaxies.
\end{abstract}

Keywords. telescopes, submillimeter, techniques: high angular resolution, techniques: interferometric

\section{Introduction to ALMA}

The Atacama Large Millimeter/Submillimeter Array, ALMA, is the largest new facility for long wavelength astronomy. It will be a large array of telescopes on a high, dry plain in the Atacama desert of northern Chile. ALMA is a joint venture of the European Southern Observatory (ESO) and Spain, the National Radio Astronomy Observatory (NRAO) of Associated Universities Inc., funded by the U.S. National Science Foundation with support from the National Research Council of Canada, and the National Institutes of Natural Sciences of Japan with the Academia Sinica of Taiwan, in cooperation with the Republic of Chile. The total cost of ALMA, based on the rebaseline of the project completed in late 2005, is $\sim 1$ billion current euros. ALMA will consist of up to $6412-\mathrm{m}$ antennas, an additional 12 7-m antennas for mapping extended emission (the Atacama Compact Array), and 4 enhanced 12-m antennas capable of total power observing. ALMA will ultimately cover all atmospheric windows at wavelengths from $3 \mathrm{~mm}$ to $350 \mu \mathrm{m}$.

ALMA is currently under construction at Llano de Chajnantor, near the historic town of San Pedro de Atacama. It shares the site with several existing and future millimeter and submillimeter facilities. Construction highlights of the past year include the completion of a road from the Operations Support Facility at $2900 \mathrm{~m}$ to the telescope site that wide enough to transport the antennas. The Array Operations Site Technical Building is nearly complete at the 5000 meter level $(16,570 \mathrm{ft})$, and is the largest steel-frame building in the world at this altitude. The ALMA Test Facility, an interferometer composed of two antenna prototypes, is being tested at the VLA site. Antenna contracts have been awarded by the partners, and the first 12-m antenna is due in Chile in 2007.

\section{ALMA science}

ALMA will be a mighty leap forward in capability, with an order of magnitude gain in sensitivity over current millimeter arrays in the northern hemisphere, and the potential

$\dagger$ Present address: ALMA, 40 El Golf, Piso 18, Las Condes, Santiago, Chile. 
for two orders of magnitude gain in imaging resolution and fidelity, at a high, dry site suited to submillimeter observing. The ALMA Level-One design goals exemplify the types of projects that will drive this transformational instrument. These goals are: $(i)$ to detect $\mathrm{CO}$ in an $\mathrm{L}^{*}$ galaxy at $z=3 ;($ ii $)$ to image molecular lines in a protoplanetary disk with a resolution of $1 \mathrm{AU}$ out to a distance of $150 \mathrm{pc}$; and (iii) to obtain high fidelity imaging at $0^{\prime \prime} .1$ to match $H S T$, JWST, or AO imaging. ALMA will allow us to study the gas in 'normal' galaxies at redshifts at which galaxies should be significantly different from the present, in the 'Gas Ages'. Closer to home, ALMA will enable study of the birth of planetary systems, allowing imaging of the nearest protoplanetary disks with $1 \mathrm{AU}$ spatial resolution and sufficient spectral resolution to resolve motions on planet scales within a gaseous protoplanetary disk.

The science capabilities of ALMA span all fields of astronomy.

- ALMA and planet formation. At its highest resolution of 5 mas, ALMA will easily detect thermal dust continuum emission from protoplanetary or debris disks out to the nearest star clusters at distances of $150 \mathrm{pc}$. It will be able to image the thermal emission from young Jupiter-sized protoplanets out to 100 pc.

- ALMA and molecular gas. ALMA will image emission from lines of the over 140 molecules currently detected in space, a list that includes organic molecules of biological interest, including amino acids. In addition to astrochemistry, studies of molecular abundances and excitation in interstellar clouds will provide diagnostics for interstellar physics, from shocks to gas dynamics, allowing the study of star formation and the feedback of star formation on molecular clouds.

- ALMA and nearby galaxies. ALMA will image molecular clouds in galaxies to give us a new view of galactic structure, galaxy interactions and galaxy mergers, and star formation on galactic scales. ALMA will probe the gas dynamics within a few parsecs of supermassive black holes in the centers of galaxies. At its highest resolution, ALMA can directly resolve structures of $50 \mathrm{AU}(\sim 600$ Schwarzschild radii) in Sgr A*.

- ALMA and the solar system. The Submillimeter Array in Hawaii recently detected the thermal emission from Pluto and Charon: while Charon's surface is the expected $50 \mathrm{~K}$ for this distance from the sun, Pluto's temperature is a chilly $38 \mathrm{~K}$; sublimation of surface ice is suspected. With its unprecedented spatial resolution and sensitivity, ALMA will be able to image surface features on the outer planets, in addition to asteroids, dwarf planets and icy Kuiper belt objects. ALMA will easily detect and perhaps even resolve the most distant object known in the solar system, UB313. The composition, structure, and kinematics of planetary atmospheres will also be within ALMA's reach.

- ALMA and the distant universe. ALMA will be a unique eye on the early universe. Actively star-forming galaxies, such as Arp 220, can be detected easily in CO to $z=10$. Images of gas and dust emission will allow us to study many properties of primeval galaxies, such as structure, dynamical masses, and gas masses. While the Hubble Deep field contains many nearby galaxies and few distant ones, the ALMA Deep Field is the opposite: to a given brightness, simulations show that galaxies beyond $z=1.5$ are a hundred times more numerous than nearby galaxies.

\section{When can I use ALMA?}

After the first antenna arrives in Chile in 2007, there will be an extended period of system integration, verification, and commissioning. 'Early Science', when ALMA is first opened to the community, is scheduled to begin in 2010, when the array has $12-16$ antennas. ALMA reaches full capability, with all antennas and receiver bands, in 2012. More ALMA information and an ALMA Cam are available at <http://www.alma.cl>. 